\title{
Effect of Bisphosphonate on Osteoclast of Bone
}

\author{
Sarah Ralte*, Asima Bhattacharyya \\ Department of Anatomy, North Eastern Indira Gandhi Regional Institute of Health and Medical Sciences \\ (NEIGRIHMS), Meghalaya, India \\ Email: "sarahzoremi@gmail.com
}

Received 17 May 2014; revised 20 June 2014; accepted 3 July 2014

Copyright (C 2014 by authors and Scientific Research Publishing Inc.

This work is licensed under the Creative Commons Attribution International License (CC BY).

http://creativecommons.org/licenses/by/4.0/

(c) () Open Access

\begin{abstract}
Bisphosphonates are synthetic analogues of naturally occurring pyrophosphate molecule and are potent inhibitors of osteoclastic bone resorption. Bisphosphonates bind to hydroxyapatite crystals with high affinity and after incorporation by osteoclasts, the primary target cell, it inhibits osteoclastic bone resorption. The anti-resorptive effect has been shown to occur in organ culture as well as in-vivo, but the precise mechanism by which it exerts its bone resorbing effect is not yet fully understood. In vitro and in vivo studies have demonstrated that zoledronate is a more potent inhibitor of osteoclasts than earlier bisphosphonates. Bisphosphonates have now emerged as a leading therapeutic agent for the treatment of hypercalcaemia of malignancy, bone metabolic diseases, Paget's disease and postmenopausal osteoporosis.
\end{abstract}

\section{Keywords}

Osteoclast, Bone Resorption, Bisphosphonate, Mechanism of Action

\section{Introduction}

The effect of various drugs has been studied in the past on the metaphysis of long bone, a site of active bone turnover with high number of osteoblasts, osteoprogenitor cells and osteoclasts [1]-[7]. Osteoclasts, the bone resorbing cells, erode mineralized bone by secreting acids and lysosomal enzymes. The increased activation of osteoclasts results in disruption of normal bone remodelling, wherein, the equilibrium between bone resorption and bone formation is shifted towards increased bone resorption [8]. Bisphosphonates are carbon substituted pyrophosphate analogues having a P-C-P bond that binds avidly to the bone mineral, hydroxyapatite crystal [9] [10]. Their P-C-P backbone structure resembles pyrophosphate, which contains oxygen instead of a carbon atom. The P-C-P structure allows a great number of possible variations either by changing the two lateral chains on the ${ }^{*}$ Corresponding author. 
carbon or by esterifying the phosphate groups. Binding to the bone mineral is enhanced when $\mathrm{R}^{1}$ is a hydroxyl. The $\mathrm{R}^{2}$ side chain defines individual bisphosphonates and determines their pharmacological activity. Depending on the structure of the two side chains $\mathrm{R}^{1}$ and $\mathrm{R}^{2}$ attached to the central carbon atom, bisphosphonates have been classified accordingly. The first generation non-nitrogen containing bisphosphonates etidronate, clodronate and tiludronate was introduced clinically more than 30 years ago. This was followed by the development of more potent second and third generation nitrogen containing bisphosphonates. The second generation bisphosphonates such as pamidronate and alendronate have an aliphatic side chain containing a single nitrogen atom while the third generation include risedronate and zoledronate, an imidazole derivative containing a second nitrogen atom in the ring structure and are the most potent bisphosphonate known to date [11].

Bisphosphonates were initially used in various industrial procedures as anticorrosive or antiscaling agents and as complexing agents in textile, fertilizers and oil industries [12]. Fleisch et al. [9], [13] were the first to report that they can effectively control calcium phosphate dissolution in vitro, as well as mineralization and bone resorption in vivo. They are now acknowledged as one of the most potent and effective inhibitors of bone resorption in skeletal diseases involving excessive osteoclast-mediated bone resorption, such as hypercalcemia of malignancy, local osteolytic hyperclacemia, Paget's disease and postmenopausal osteoporosis [14] [15]. Reitsma et al. [16] reported that due to the strong affinity for bone mineral, bisphosphonates accumulate in the mineralized bone matrix for several weeks and are localized specifically at the edge of trabeculae and at the interface of osteoid mineralized bone.

\section{Past Animal Studies on the Effect of Bisphosphonate on Bone}

Various studies have been conducted in the past to find out the effects of bisphosphonates on the metaphysis of rats. Fleisch et al. in 1969 first described the effects of bisphosphonates containing the P-C-P bond, sodium dichloromethylene bisphosphonate and sodium methylene bisphosphonate on the dissolution of apatite crystals in vitro and on parathyroid extract (PTE) induced bone resorption in tissue culture and in living rats. A monophosphonate, sodium pentane-1-phosphonate, containing only a single C-P bond, along with pyrophosphate and polyphosphate were used as controls. Addition of the bisphosphonates and pyrophosphate to apatite crystals resulted in reduction of the rate at which calcium and phosphate entered the solution while the monophosphate had no such effect. In the next experiment, calvaria from three day old mice were cultured individually for five days and PTE was added which induced detectable resorption. It was observed that bone resorption was inhibited by both the bisphosphonate at very low concentration, while rest of the phosphates was not inhibitory even at high concentration. Hypercalcemia in rats given prior treatment with PTE for several days was reversed by administration of bisphosphonates, indicating that the bisphosphonates reduced bone resorption [9]. Short term treatment of rats with clodronate, showed that at doses of $10 \mathrm{mg} / \mathrm{kg} /$ day, the newly formed part of the metaphysis took on a cylindrical shape and at $30 \mathrm{mg} / \mathrm{kg} /$ day there was a further increase in bone density and more pronounced cylindrical shape [17]. Miller and Jee [18] studied the ultrastructural effects of clodronate $\left(\mathrm{Cl}_{2} \mathrm{MBP}\right)$ on the bone cells in 20 young growing male rats after administration of $20 \mathrm{mg} / \mathrm{kg} /$ day of clodronate subcutaneously for a period of ten days. In the $\mathrm{Cl}_{2} \mathrm{MBP}$ treated animals, the amount of trabecular bone in the tibial metaphysis was greatly increased. Slender bone trabeculae, characteristic of the primary spongiosa was seen to extend towards the diaphysis. Numerous, large trabeculae in the secondary spongiosa were seen extending further towards the diaphyseal region. In the control metaphyseal bone, large areas of bone surface were covered with plump osteoblasts. Osteoclasts were usually found at the ends of bone spicules. In $\mathrm{Cl}_{2} \mathrm{MBP}$ treated bone, osteoblasts were more abundant in the primary spongiosa than secondary spongiosa, which was seen to be greatly enlarged. Numerous large osteoclasts were found on trabecular bone surfaces. Most of these osteoclasts were large in size with increased number of nuclei, appearing as bags of nuclei hanging from bone spicules. At the ultrastructural level, the most conspicuous feature of bone was the presence of vast resorption areas covering most of the bone surfaces. These resorption area contained resorption pits or Howship's lacunae, which were small, shallow and had poorly defined margins as compared to the control group. Single osteoclasts were often found to be covering several of the small resorption pits. Clear zones were seen to be in close contact with the bone surface and often appeared to be very extensive in $\mathrm{Cl}_{2} \mathrm{MBP}$ treated osteoclasts, covering large areas of bone surface. Ruffled borders found on these osteoclasts were generally smaller and less extensive than those encountered in the control group. Cytoplasmic vacuole-likestructures, commonly associated with normal ruffled borders, were smaller and less in number. The extent of stained material beneath the osteoclasts ruffled border region adjacent to the bone was observed to be less in the $\mathrm{Cl}_{2} \mathrm{MBP}$ treated animals as compared to controls, sug- 
gesting that the stained material was most probably bone matrix which had been partially demineralized by osteoclastic action. The authors concluded that the extent of bone surface actively being resorbed per osteoclast appeared to be less in the $\mathrm{Cl}_{2} \mathrm{MBP}$ treated animals and correlated well with the decreases observed by electron microscope in the extent of the ruffled borders on these cells. These findings accounted for the small, shallow, poorly defined resorption pits characteristic of bisphosphonate treated bone. Increase in osteoclast population as well as increase in bone surface resorption areas was not necessarily tantamount with an absolute increase in bone resorption, suggesting that there remained several possibilities as to the exact mechanism of action of this compound. Regardless of the exact mechanism of action of clodronate on osteoclasts and bone resorption, it was quite clear that clodronate had considerable effects on osteoclast populations, morphology and its function.

Ito et al. in 1999 conducted the ultrastructural and cytochemical studies on cell death of osteoclasts induced by bisphosphonate treatment on rat skeleton. Histological, cytochemical and ultrastructural features of osteoclasts undergoing apoptosis were studied in the femur and tibia of rats treated with a third generation bisphosphonate. After the bisphosphonate administration, osteoclast decreased significantly in number. Initially they were devoid of ruffled border and were detached from the bone surface. In such osteoclasts, the golgi apparatus was degraded or dispersed in the cytoplasm. Later, osteoclasts revealed typical features of apoptosis, with pyknotic nuclei showing condensation and magnification of heterochromatins and DNA fragmentation. They were often convoluted giving rise to apoptotic bodies. Enlargement and fusion of nuclear envelopes and subsequent disruption leading to leakage of nuclear contents into the cytoplasm were observed in osteoclasts in the late stage of apoptosis. These osteoclasts as well as apoptotic bodies were seen to be surrounded by cytoplasmic processes of macrophages, which often contained degenerated cytoplasmic fragments of osteoclasts. Apoptotic osteoclasts migrating into or present in capillaries were also observed in some areas. Bisphosphonate induced apoptosis of osteoclasts, which was characterized by ultrastructural changes of the nucleus, typical of apoptosis accompanied by degradation of cell organelles. The authors reported that most of the damaged osteoclasts were eliminated by macrophages while some escaped into the blood vessels [19].

The effect of protein prenylation (geranylgeranylation) and its inhibition by nitrogen containing bisphosphonates and geranylgeranyltransferase-I inhibitor-298 (GGTI-298) was studied by Coxon and his co-workers [20] in chick and rabbit osteoclasts in vitro. The nitrogen containing bisphosphonates such as risedronate, zoledronate, ibandronate, alendronate and pamidronate presented the incorporation of ${ }^{14} \mathrm{C}$-mevalonate into prenylated (farnesylated and geranylgeranylated) proteins in purified rabbit osteoclasts. It was suggested that the inhibitory effect of nitrogen containing bisphosphonates on bone resorption resulted largely from the loss of geranylgeranylated proteins rather than loss of farnesylated proteins in osteoclasts. Administration of GGTI-298, a specific inhibitor of geranylgeranyl transferase-I inhibited protein geranylgeranylation in purified rabbit osteoclasts, prevented osteoclast formation in murine bone marrow cultures, disrupted the osteoclasts cytoskeleton, inhibited bone resorption and induced apoptosis in isolated chick and rabbit osteoclasts in vitro. While concentrations of FTI-277, a specific inhibitor of farnesyl transferase prevented protein farnesylation in purified rabbit osteoclasts with little effect on osteoclast morphology or apoptosis and did not inhibit bone resorption. The results highlighted the fundamental importance of geranylgeranylated proteins in osteoclast formation and function and its inhibition by bisphosphonates explained the molecular mechanism of action of nitrogen containing bisphosphonate drugs in osteoclasts. Alakangas et al. [21] investigated the possible interference of alendronate with guanosine triphosphatase (GTPase) proteins involved in regulation of vesicle transport in isolated rat osteoclasts. Mature osteoclasts were mechanically harvested from endosteal surface of long bones of 1 - 2 days old rat pups. Cells were then cultured on to control bone slices and alendronate as well as clodronate coated bone slices and examined under electron microscope. The number and size of the individual resorption pits were decreased on the alendronate covered bone slices. The shapes of the resorption pits on both bisphosphonates covered bone slices differed from those seen on the control bone slices. Pits on control slices typically had a complex shape, whereas those on the bisphosphonate covered bones were simple and round. Osteoclasts in the control and alendronate groups were examined by transmission electron microscope. Osteoclasts in the control groups were tightly attached to the bone surface and showed well defined sealing zones surrounding the ruffled borders which typically penetrated into the bone. Lucent vesicles and functional secretory domains (FSDs) with numerous microvilli on top of the osteolasts were observed. In alendronate treated osteoclasts, ruffled borders were poorly organized and detached from the bone surface. Sealing zones appeared incomplete and often detected at only one boundary of the poorly formed ruffled border. FSDs showed less microvilli. Numerous small, lucent vesicles were detected in the bone facing cytoplasm and long, tubular vesicles containing electron dense material were observed in alendronate treated osteoclasts. Immunofluorescent staining for tartrate resistant acid phos- 
phatase (TRAP) showed increased TRAP activity. Accumulation of TRAP, defective FSDs and the appearance of electron dense material containing vesicles in intracellular compartments of alendronate treated osteoclasts indicated that the transcytotic route was disturbed by alendronate. It was suggested that the nitrogen containing bisphosphonate alendronate inhibited osteoclast mediated bone resorption through inhibition of the mevalonate pathway resulting in impaired protein prenylation and affecting the function of small GTPase in osteoclasts involved in intracellular vesicle transport. Lehenkari and his co-workers [22] studied the effects of alendronate, clodronate, and the clodronate metabolite Adenosine-5'-( $\beta, \gamma$-dichloromethylene) triphosphate $\left(\mathrm{AppCCl}_{2} \mathrm{P}\right)$ on isolated mitochondria, mitochondrial fractions, and mitochondrial membrane potential in isolated human osteoclasts. It was found that $\mathrm{AppCCl}_{2} \mathrm{P}$ inhibited mitochondrial oxygen consumption by mechanisms that involve competitive inhibition of the adenine nucleotide translocase (ADP/ATP translocase). Alendronate or the native form of clodronate did not have any immediate effect on mitochondria while longer treatment with liposome-encapsulated clodronate caused collapse of the mitochondrial membrane potential, although prominent apoptosis was a late event. Inhibition of the ADP/ATP translocase by the metabolite $\mathrm{AppCCl}_{2} \mathrm{p}$ was suggested to be the likely route by which clodronate caused osteoclast apoptosis and inhibited bone resorption. Long term administration of pamidronate significantly reduced osteoclastic bone resorption and effectively increased trabecular bone volume in ovariectomized induced acute estrogen deficiency in mature rats [23].

Recently, Ralte S et al. [24] examined the effects of short-term administration of zoledronate, a third generation bisphosphonate, on the metaphysis of the proximal end of tibia in twenty days old male albino rats. Zoledronate ( $2.8 \mu \mathrm{g} / \mathrm{kg}$ body weight), was given subcutaneously daily for eleven days. The animals were sacrificed; tibiae were dissected out and decalcified in ethylene di-amine tetra-acetic acid (EDTA). Seven micron thick, serial longitudinal paraffin sections were stained with Hematoxylin and Eosin and examined under Zeiss light microscope and Image Pro-Express Analyzer. In zoledronate treated rats, a significant increase $(p<0.05)$ in the number of osteoclasts was observed both in the regions of primary spongiosa (zoledronate treated: $6.41 \pm$ $0.30 / \mathrm{mm}^{2}$, control: $2.90 \pm 0.28 / \mathrm{mm}^{2}$ ) and secondary spongiosa (zoledronate treated: $49.58 \pm 0.84 / \mathrm{mm}^{2}$, control: $\left.31.81 \pm 2.02 / \mathrm{mm}^{2}\right)$ along with a significant increase $(\mathrm{p}<0.05)$ in the length of the metaphyseal region as compared to control group. The number of nuclei per osteoclast and area of osteoclast also showed a significant increase ( $\mathrm{p}<0.001 ; \mathrm{p}<0.05$ respectively) following the uptake of zoledronate. We observed that the siginificant increase in the number and size of osteoclast appeared to be a paradox against the antiresorptive actions of zoledronate; indicating that the osteoclast is most probably the site of action of zoledronate resulting in decreased osteoclastic activity which accounted for the great increase in the number of osteoclasts and marked bone formation in our study (Figure 1).

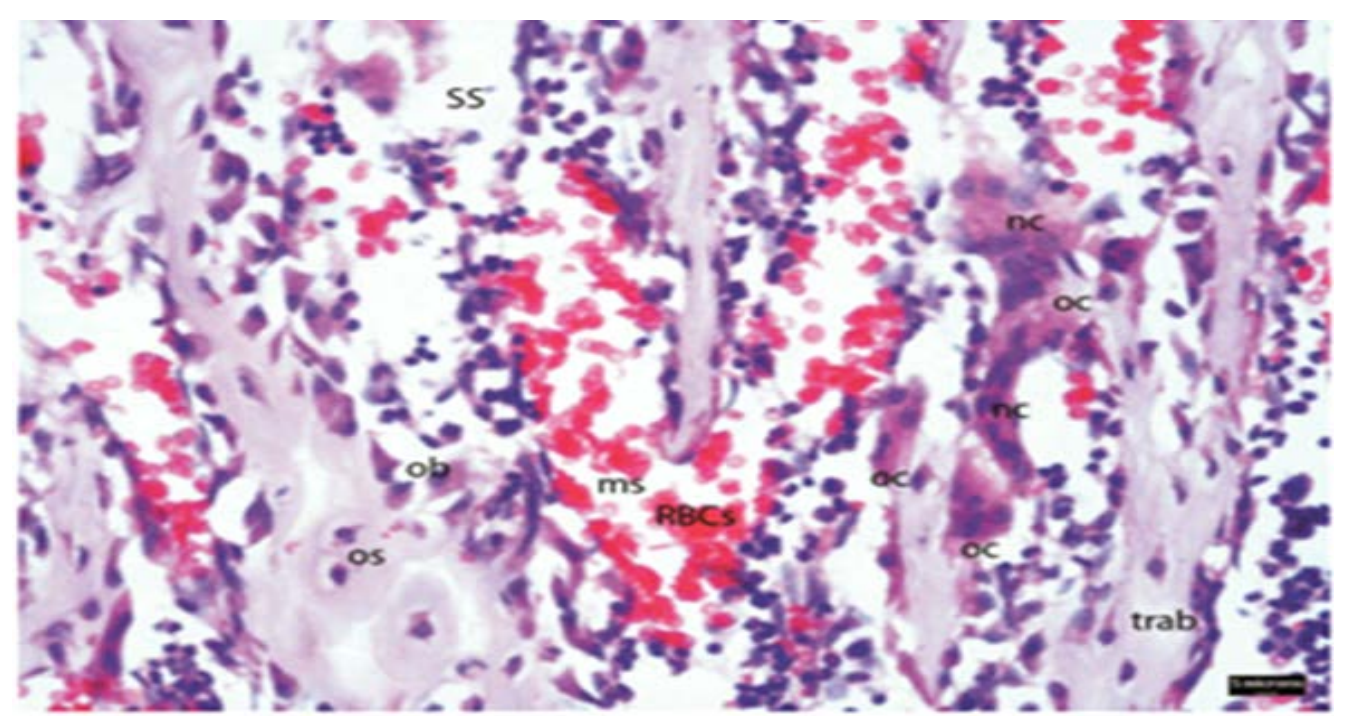

Figure 1. Photomicrograph of proximal tibial metaphysis of rat from zoledronate treated group, showing large osteoclasts. Hematoxylin \& Eosin (H \& E 200×). (Abbreviation: oc-osteoclast; os-osteocyte; obosteoblast; nc-nucleus; trab-trabeculae; SS-secondary spongiosa; ms-medullary space, RBCs-red blood corpuscles). 


\section{Proposed Mechanism of Actions}

Since the discovery of their effects on biological tissues in 1968, much effort has been made in understanding the mechanism of action of the bisphosphonates. Fleisch (1969) published the first paper on the inhibitory effect of bisphosphonates on the dissolution of hydroxyapatite crystals in vitro accounting for their selective action on bone tissues. They suggested that bisphosphonates have two fundamental biological effects, inhibition of calcification by retarding the formation of calcium phosphate salts, a physicochemical process and inhibition of bone resorption at a cellular level.

Since then various mechanisms of action have been cited by different authors and are as follows:

- Inhibition of pathological calcification [10].

- Incorporation of bisphosphonate into the osteoclast at the ruffled border [18].

- Inhibition of recruitment, activation and differentiation of osteoclast precursors [25].

- Interference with chemotaxis and attachment of osteoclast to bone [26].

- Alteration to the osteoclast cytoskeleton [27].

- Inhibition of osteoclastic activity by osteoblast mediated signals [28].

- Suppression of mature osteoclast function [11].

- Disruption of ruffled border formation and actin ring filament in osteoclast [29].

- Direct or indirect stimulation of osteoclast apoptosis [30].

- Reduced proton-pump ATPase ( $\mathrm{H}^{+}$ATPase) expression in osteoclast [23].

- Reduced production of cytokines like interleukin-6 [31].

- Direct cytostatic and cytolytic activity [32].

- Inhibition of tumor cell dissemination, invasion and adhesion to the bone matrix [33].

- Defective intracellular vesicle transport in osteoclast which in trun prevents osteoclast from forming a tight scaling zone or ruffled border, required for bone resorption [20].

- Recently, Lehenkari et al. [22] reported that bisphosphonates are metabolized intracellularly to cytotoxic, nonhydrolyzable inactive analogues of adenosine triphosphate by inhibiting mitochondrial ADP/ATP translocase resulting in osteoclastic apoptosis.

The precise molecular mechanism of action of bisphosphonates leading to the inhibition of osteoclastic activity still however remains unclear to date.

\section{References}

[1] Simmons, D.J. (1963) Cellular Changes in the Bones of Mice as Studied with Tritiated Thymidine and the Effects of Estrogen. Clinical Orthopaedics and Related Research, 26, 176-189.

[2] Young, M.H. and Crane, W.A. (1964) Effect of Hydrocortisone on the Utilization of Tritiated Thymidine for Skeletal Growth in the Rat. Annals of the Rheumatic Diseases, 23, 163-168. http://dx.doi.org/10.1136/ard.23.2.163

[3] Russell, R.G., Kisling, A.M., Casey, P.A., Fleisch, H., Thornton, J., Schenk, R. and Williams, D.A. (1973) Effect of Diphosphonates and Calcitonin on the Chemistry and Quantitative Histology of Rat Bone. Calcified Tissue Research, 11, 179-195. http://dx.doi.org/10.1007/BF02547218

[4] Stutzer, A., Fleisch, H. and Trechsel, U. (1988) Short- and Long-Term Effects of a Single Dose of Bisphosphonates on Retinoid-Induced Bone Resorption in Thyroparathyroidectomized Rats. Calcified Tissue International, 43, $294-299$. http://dx.doi.org/10.1007/BF02556639

[5] Wronski, T.J., Dann, L.M., Scott, K.S. and Crooke, L.R. (1989) Endocrine and Pharmacological Suppressors of Bone Turnover Protect against Osteopenia in Ovariectomized Rats. Endocrinology, 125, 810-816. http://dx.doi.org/10.1210/endo-125-2-810

[6] Lin, B.Y., Jee, W.S., Ma, Y.F., Ke, H.Z., Kimmel, D.B. and Li, X.J. (1994) Effects of Prostaglandin E2 and Risedronate Administration on Cancellous Bone in Older Female Rats. Bone, 15, 489-496. http://dx.doi.org/10.1016/8756-3282(94)90272-0

[7] Turan, B., Balcik, C. and Akkas, N. (1997) Effect of Dietary Selenium and Vitamin E on Biochemical Properties of Rabbit Bone. Clinical Rheumatology, 16, 441-449. http://dx.doi.org/10.1007/BF02238935

[8] Fleisch, H. (1998) Bisphosphonates: Mechanisms of Action. Endocrine Reviews, 19, 80-100. http://dx.doi.org/10.1210/edrv.19.1.0325

[9] Fleisch, H., Russell, R.G. and Francis, M.D. (1969) Diphosphonates Inhibit Hydroxyapatite Dissolution in Vitro and Bone Resorption in Tissue Culture and in Vivo. Science, 165, 1262-1264. http://dx.doi.org/10.1126/science.165.3899.1262 
[10] Francis, M.D., Graham, R., Russell, R.G. and Fleisch, H. (1969) Diphosphonates Inhibit Formation of Calcium Phosphate Crystals in Vitro and Pathological Calcification in Vivo. Science, 165, 1264-1266. http://dx.doi.org/10.1126/science.165.3899.1264

[11] Green, J.R., Muller, K. and Jaeggi, K.A. (1994) Preclinical Pharmacology of CGP 42’446, a New, Potent, Heterocyclic Bisphosphonate Compound. Journal of Bone and Mineral Research, 9, 745-751. http://dx.doi.org/10.1002/jbmr.5650090521

[12] Menschutkin, N. (1865) Ueber die Einwirkung des Chloracetyles auf Phosphorige Saure. Justus Liebigs Annalen der Chemie, 133, 317-320. http://dx.doi.org/10.1002/jlac.18651330307

[13] Fleisch, H., Russell, R.G., Bisaz, S., Casey, P.A. and Muhlbauer, R.C. (1968) The Influence of Pyrophosphate Ana-Logues (Diphosphonates) on the Precipitation and Dissolution of Calcium Phosphate in Vitro and in Vivo. Calcified Tissue Research, 2, 10-10A. http://dx.doi.org/10.1007/BF02065192

[14] Ernst, D.S., Brasher, P., Hagen, N., Paterson, A.H., MacDonald, R.N. and Bruera, E. (1997) A Randomized, Controlled Trial of Intravenous Clodronate in Patients with Metastatic Bone Disease and Pain. Journal of Pain and Symptom Management, 13, 319-326. http://dx.doi.org/10.1016/S0885-3924(97)00075-4

[15] Rossini, M., Gatti, D., Girardello, S., Braga, V., James, G. and Adami, S. (2000) Effects of Two Intermittent Alendronate Regimens in the Prevention or Treatment of Postmenopausal Osteoporosis. Bone, 27, 119-122. http://dx.doi.org/10.1016/S8756-3282(00)00291-X

[16] Reitsma, P.H., Teitelbaum, S.L., Bijvoet, O.L. and Kahn, A.J. (1982) Differential Action of the Bisphosphonates (3-Amino-1-hydroxypropylidene)-1, 1-Bisphosphonate (APD) and Disodium Dichloromethylidene Bisphosphonate $\left(\mathrm{Cl}_{2} \mathrm{MDP}\right)$ on Rat Macrophage-Mediated Bone Resorption in Vitro. Journal of Clinical Investigation, 70, 927-933. http://dx.doi.org/10.1172/JCI110704

[17] Schenk, R., Merz, W.A., Muhlbauer, R., Russell, R.G. and Fleisch, H. (1973) Effect of Ethane-1-hydroxy-1, 1-diphosphonate (EHDP) and Dichloromethylene Diphosphonate $\left(\mathrm{Cl}_{2} \mathrm{MDP}\right)$ on the Calcification and Resorption of Cartilage and Bone in the Tibial Epiphysis and Metaphysis of Rats. Calcified Tissue Research, 11, 196-214. http://dx.doi.org/10.1007/BF02547219

[18] Miller, S.C. and Jee, W.S. (1979) The Effect of Dichloromethylene Diphosphonate, a Pyrophosphate Analog, on Bone and Bone Cell Structure in the Growing Rat. Anatomical Record, 193, 439-462. http://dx.doi.org/10.1002/ar.1091930309

[19] Ito, M., Amizuka, N., Nakajima, T. and Ozawa, H. (1999) Ultrastructural and Cytochemical Studies on Cell Death of Os-Teoclasts Induced by Bisphosphonate Treatment. Bone, 25, 447-452. http://dx.doi.org/10.1016/S8756-3282(99)00197-0

[20] Coxon, F.P., Helfrich, M.H., Van’t Hof, R., Sebti, S., Ralston, S.H., Hamilton, A. and Rogers, M.J. (2000) Protein Geranylgeranylation Is Required for Osteoclast Formation, Function, and Survival: Inhibition by Bisphosphonates and GGTI-298. Journal of Bone and Mineral Research, 15, 1467-1475. http://dx.doi.org/10.1359/jbmr.2000.15.8.1467

[21] Alakangas, A., Selander, K., Mulari, M., Halleen, J., Lehenkari, P., Monkkonen, J., Salo, J. and Vaananen, K. (2002) Alendronate Disturbs Vesicular Trafficking in Osteoclasts. Calcified Tissue International, 70, 40-47. http://dx.doi.org/10.1007/s002230010047

[22] Lehenkari, P.P., Kellinsalmi, M., Napankangas, J.P., Ylitalo, K.V., Monkkonen, J., Rogers, M.J., Azhayey, A., Vaananen, H.K. and Hassinen, I.E. (2002) Further Insight into Mechanism of Action of Clodronate: Inhibition of MitoChondrial ADP/ATP Translocase by a Nonhydrolyzable, Adenine-Containing Metabolite. Molecular Pharmacology, 61, 1255-1262. http://dx.doi.org/10.1124/mol.61.5.1255

[23] Mayahara, M. and Sasaki, T. (2003) Cellular Mechanism of Inhibition of Osteoclastic Resorption of Bone and Calcified Cartilage by Long-Term Pamidronate Administration in Ovariectomized Mature Rats. Anatomical Record Part A, 274, 817-826. http://dx.doi.org/10.1002/ar.a.10092

[24] Ralte, S., Khatri, K. and Nagar, M. (2011) Short-Term Effects of Zoledronate on the Histomorphology of Osteoclast in Young Albino Rats. Annals of Anatomy, 193, 509-515. http://dx.doi.org/10.1016/j.aanat.2011.03.009

[25] Hughes, D.E., MacDonald, B.R., Russell, R.G. and Gowen, M. (1989) Inhibition of Osteoclast-Like Cell Formation by Bisphosphonates in Long-Term Cultures of Human Bone Marrow. Journal of Clinical Investigation, 83, 1930-1935. http://dx.doi.org/10.1172/JCI114100

[26] Flanagan, A.M. and Chambers, T.J. (1991) Inhibition of Bone Resorption by Bisphosphonates: Interactions between Bisphosphonates, Osteoclasts, and Bone. Calcified Tissue International, 49, 407-415. http://dx.doi.org/10.1007/BF02555852

[27] Sato, M., Grasser, W., Endo, N., Akins, R., Simmons, H., Thompson, D.D., Golub, E. and Rodan, G.A. (1991) Bisphosphonate Action. Alendronate Localization in Rat Bone and Effects on Osteoclast Ultrastructure. Journal of Clinical Investigation, 88, 2095-2105. http://dx.doi.org/10.1172/JCI115539 
[28] Vitte, C., Fleisch, H. and Guenther, H.L. (1996) Bisphosphonates Induce Osteoblasts to Secrete an Inhibitor of Osteoclast-Mediated Resorption. Endocrinology, 137, 2324-2333.

[29] Murakami, H., Takahashi, N., Sasaki, T., Udagawa, N., Tanaka, S., Nakamura, I., Zhang, D., Barbier, A. and Suda, T. (1995) A Possible Mechanism of the Specific Action of Bisphosphonates on Osteoclasts: Tiludronate Preferentially Affects Polarized Osteoclasts Having Ruffled Borders. Bone, 17, 137-144. http://dx.doi.org/10.1016/S8756-3282(95)00150-6

[30] Hughes, D.E., Wright, K.R., Uy, H.L., Sasaki, A., Yoneda, T., Roodman, G.D., Mundy, G.R. and Boyce, B.F. (1995) Bisphosphonates Promote Apoptosis in Murine Osteoclasts in Vitro and in Vivo. Journal of Bone and Mineral Research, 10, 1478-1487. http://dx.doi.org/10.1002/jbmr.5650101008

[31] Derenne, S., Amiot, M., Barille, S., Collette, M., Robillard, N., Berthaud, P., Harousseau, J.L. and Bataille, R. (1999) Zoledronate Is a Potent Inhibitor of Myeloma Cell Growth and Secretion of IL-6 and MMP-1 by the Tumoral Environment. Journal of Bone and Mineral Research, 14, 2048-2056. http://dx.doi.org/10.1359/jbmr.1999.14.12.2048

[32] Jagdev, S.P., Croucher, P.I. and Shipman, C.M. (2000) Taxol and Zoledronate Induce Apoptosis in Breast Cancer Cells in Vitro: Evidence for Action via Inhibition of Mevalonate Pathway. Bone, 26, S30.

[33] Biossier, S., Ferreras, M., Peyruchaud, O., Magnetto, S., Ebetino, F.H., Colombel, M., Delmas, P., Delaissé, J.M. and Clézardin, P. (2000) Biphosphonates Inhibit Breast and Prostrate Carcinoma Cell Invasion, an Early Event in the Formation of Bone Metastases. Cancer Research, 60, 2949-2954. 